\title{
Frequency and predictors of access site complications of the transfemoral diagnostic coronary procedures
}

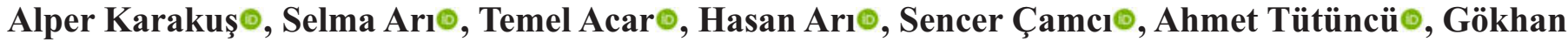 \\ Özmen $\odot$, Mehmet Melek॰, Mehmet Demir॰
}

Department of Cardiology, University of Health Sciences, Bursa Yüksek Ihtisas Training and Research Hospital, Bursa, Turkey

\begin{abstract}
Objectives: Access site complications (ASC) remain important and common adverse events after coronary angiography. The aim of this study was to evaluate the frequency and predictors of ASC in patients undergoing diagnostic coronary angiography (DCA) via the femoral artery.

Methods: In this prospective analytical cross sectional study, DCA was performed in 3972 patients by transfemoral approach. The femoral access site hemostasis was obtained by manual compression in all patients. Demographic characteristics of the patients, size of the arterial sheaths, duration of compression time, patients blood pressure, medications, hemoglobin and platelet levels were recorded. All patients were evaluated for ASC (pseudoaneurysm, bleeding, dissection, hematoma). The ASC rate was calculated and ASC predictors were determined by multivariate analysis.

Results: The ASC rate was $1.3 \%$ (53 of 3972 patients). The complications were more frequent in female (female: 29 of 53 [54.7\%] vs male: 24 of 53 [45.3\%]; $p=0.007)$ and chronic renal failure (CRF) patients $(103$ of $3919[2.6 \%]$ vs 4 of 53 [7.5\%]; $p=0.02)$. Hemoglobin level was lower $(13.40 \pm 1.73$ vs $12.86 \pm 1.75 ; p=$ $0.02)$ and manual compression time was longer $(9.19 \pm 3.28 \mathrm{~min}$ vs $14.53 \pm 6.47 \mathrm{~min} ; p<0.001)$ in the ASC group. Clopidogrel (188 of 3919 [4.8\%] vs 7 of $53[13.2 \%] ; p=0.005)$ and low molecular weight heparin (LMWH) using were more frequent (37 of 3919 [0.9\%] vs 7 of $53[13.2 \%] ; p<0.001$ ) in the ASC group. According to multivariate analysis; female gender; OR: 2.13, 95\% CI: 1.14-3.99 $(p=0.017)$, presence of CRF; OR: $3.15,95 \%$ CI: 1.06-9.29 ( $p=0.038)$, manual compression time; OR: $1.23,95 \%$ CI: $1.17-1.30(p<0.001)$ and LMWH using; OR: 15.68, 95\% CI: 6.05-40.61 $(p<0.001)$ were predictors of ASC.

Conclusions: The incidence of femoral ASC rate was $1.3 \%$ in patients with DCA. Female gender, presence of CRF, using LMWH and long manual compression time were predictors of ASC.

Keywords: Vascular access devices, coronary angiography, femoral artery, complication
\end{abstract}

O ver decades, percutaneous coronary angiography has been standard diagnostic strategy for coronary artery disease [1]. Access site complications (ACSs) after diagnostic coronary angiography (DCA) include local site and retroperitoneal hematomas, significant bleeding, peripheral embolization, dissection, aneurysm, pseudoaneurysms, infection, injury to other local structures and arteriovenous fistulas $[2,3]$. The common femoral artery has long been the access site for performing coronary angiography and angioplasty. The vascular complication or bleeding risk associated with the transfemoral approach (TFA) is reported up

How to cite this article: Karakuş A, Art S, Acar T, Arl H, Camcl S, Tütüncü A, et al. Frequency and predictors of access site complications of the transfemoral diagnostic coronary procedures. Eur Res J 2020;6(6):640-646. DOI: 10.18621/eurj.707942

Address for correspondence: Hasan Arl, MD., Associate Professor, University of Health Sciences, Bursa Yüksek Ihtisas Training and Research Hospital, Department of Cardiology,Bursa,Turkey.E-mail: hasanari03@yahoo.com 
to $10 \%[4,5]$. The use of the transradial approach (TRA) is nowadays more widespread and the international guidelines have recommended radial access as a preferred approach in patients with high bleeding risk. Nevertheless, the radial access takes longer procedural time, with higher radiation exposure and cost than TFA. And also, the use of vascular closure device (VCD) for TFA has increased dramatically. The efficacy of achieving hemostasis with VCD seems similar to traditional manual compression [6]. The use of VCD for TFA, results with procedural cost increases. Additionally, the use of these devices is not without complications $[7,8]$.

The aim of this study was to identify incidence and predictors of ASCs with TFA and to determine the patients group who will have ASCs with TFA, during DCA.

\section{METHODS}

\section{Study Design and Patients}

A total of 3972 consecutive patients who underwent elective DCA by TFA over a one-year period (2015 to 2017) were enrolled in to this prospective analytical cross sectional study. The study protocol was approved by the local ethics committee. All the patients provided written informed consent. Exclusion criteria included severe sepsis, local site infection (femoral site), previous contrast allergy, severe intrinsic/iatrogenic coagulopathy (INR $>2$ ), patients DCA performed by TFA in last four week and peripheral vascular disease (iliofemoral disease). Patients who were treated with low molecular weight heparin (LMWH) within the last 12 hours were excluded from the study. Acute coronary syndrome patients, the patients who had planned to undergo ad-hoc percutaneous coronary intervention (PCI) were also excluded. No patient was excluded due to possible femoral complications (obecity, etc).

Each patient was interviewed and examined by a physician before the DCA. The clinical variables included patients' age, sex, body mass index (BMI), presence or absence of hypertension, diabetes mellitus and chronic renal failure (CRF), medications, number of intervention, blood pressure level, and hemoglobin and platelet levels were recorded. The procedural parameters included the size of the arterial sheaths, the peri- or post-procedural use of antiplatelet and anticoagulant, and the duration of compression time after sheath removal were also recorded.

Patients whose systolic blood pressure (SBP) $\geq$ $140 \mathrm{mmHg}$, or whose diastolic blood pressure (DBP) $\geq 90 \mathrm{mmHg}$ and patients who use anti-hypertensive medication were accepted as hypertension. Patients were defined as diabetic if fasting blood glucose level was $\geq 126 \mathrm{mg} / \mathrm{dL}$ blood on two consecutive measurements or if they used oral antidiabetics/insülin. Patients with an estimated glomerular filtration rate (eGFR) of $<15 \mathrm{ml} / \mathrm{min} / 1.73 \mathrm{~m} 2$, or who require hemodialysis or peritoneal dialysis treatment were defined as in CRF. eGFR was calculated with Cockcroft-Gault formula; [(140-age $) \times$ body weight $(\mathrm{kg})] /$ [72 $\times$ serum creatinine] (if women $\times 0.85$ ) [9].

\section{Coronary Angiography Procedure}

All procedures have been performed by 6 experienced (operator with annual volumes of $>75$ PCI procedures) cardiologist at our institution. Either $6 \mathrm{~F}$ or $7 \mathrm{~F}$ sheaths were introduced into the femoral artery by using standard modified Seldinger technique. After the procedure, the femoral sheath was removed in the catheter lab. Access site hemostasis was achieved by manual compression in all patients by experienced cath lab staff. Manual compression interval was defined as the time between sheath removal to hemostasis. A sand bag has been applied for 6 hours over access site after hemostasis and patients were followed for occurrence any acute complications at the Cardiology ward. VCD was not used in any patient. No patient was required to switch from TFA to TRA. Access sites was also checked before discharge by performing physician. Vascular Doppler evaluation was performed for patients with suspected ASCs. Both index in-hospital data and post-procedural 2nd week outpatient visit data regarding ASCs were recorded in all patients.

\section{Study Outcomes}

The primary endpoint was the occurring of major ASCs. The major ASCs were defined as presence of groin hematoma $>10 \mathrm{~cm}$ in diameter, aneurysm or pseudoaneurysm, arteriovenous fistula, significant bleeding, dissection and retroperitoneal hemorrhage. Significant bleeding was defined as blood loss with a 
$>3 \mathrm{~g} / \mathrm{dl}$ drop in hemoglobin or a $10 \%$ decrease in hematocrit [10]. Aneurysm, pseudoaneurysm and arteriovenous fistula were diagnosed by vascular Doppler ultrasound evaluation. Dissection was diagnosed by conventional angiographic evaluation. Retroperitoneal hemorrhage was diagnosed by computed tomography.

\section{Statistical Analysis}

The data were analyzed using the SPSS 10.0 statistics package (SPSS Inc., Chicago, Ill, USA). Continuous variables are reported as means \pm standard deviation and categorical variables are reported as percentages. Student's t test was used for comparison of normal distributed variables and Mann-Whitney U test was used for non-normally distributed variables. Categorical variables were compared by the Chi-Square test or Fisher's exact test as appropriate. Univariate and multivariate logistic regression analyses were used to determine significant predictors of ASCs. The sensitivity and specificity of compression time of after sheath removal to predict ASCs was analyzed by receiver operating characteristic (ROC) analysis. $P$ values less than 0.05 were considered significant.

\section{RESULTS}

Access site complications were observed in a total of $53(1.3 \%)$ patients. The baseline demographic, hematologic, access site characteristics and anticoagulant and antithrombotic medications of the patients with and without ASC were represented in table 1.

The female gender, presence of CRF, clopidogrel use and low molecular weight heparin (LMWH) use

Table 1. Baseline characteristics of the study patients

\begin{tabular}{|c|c|c|c|}
\hline & $\begin{array}{c}\text { Group } 1 \\
(n=3919)\end{array}$ & $\begin{array}{l}\text { Group } 2 \\
(n=53)\end{array}$ & $p$ value \\
\hline Age (years) & $59.57 \pm 11.90$ & $57.47 \pm 11.34$ & 0.20 \\
\hline Gender, n (\%) & & & 0.007 \\
\hline Male & $2482(63.3)$ & $24(45.3)$ & \\
\hline Female & $1437(36.7)$ & $29(54.7)$ & \\
\hline BMI $\left(\mathrm{kg} / \mathrm{m}^{2}\right)$ & $28.44 \pm 4.84$ & $28.10 \pm 4.89$ & 0.61 \\
\hline Hypertension, $\mathrm{n}(\%)$ & $1982(50.6)$ & $28(52.8)$ & 0.74 \\
\hline Diabetes Mellitus, n (\%) & $1172(29.9)$ & $17(32.1)$ & 0.73 \\
\hline CRF, n (\%) & $103(2.6)$ & $4(7.5)$ & 0.02 \\
\hline Systolic blood pressure (mmHg) & $141.78 \pm 22.55$ & $143.07 \pm 23.13$ & 0.68 \\
\hline Diastolic blood pressure (mmHg) & $80.28 \pm 11.43$ & $79.61 \pm 14.13$ & 0.67 \\
\hline Hemoglobin (gr/dl) & $13.40 \pm 1.73$ & $12.86 \pm 1.75$ & 0.02 \\
\hline Platelet $\left(\times 10^{3} / \mathrm{ul}\right)$ & $248.04 \pm 75.17$ & $236.67 \pm 53.46$ & 0.27 \\
\hline Manual compression time (min) & $9.19 \pm 3.28$ & $14.53 \pm 6.47$ & $<0.001$ \\
\hline Sheath Size, n (\%) & & & 0.36 \\
\hline $6 \mathrm{~F}$ & $3858(98.4)$ & $(100)$ & \\
\hline $7 F$ & $61(1.6)$ & - & \\
\hline \multicolumn{4}{|l|}{ Medication, $\mathrm{n}(\%)$} \\
\hline Aspirin & $1881(48)$ & $28(52.8)$ & 0.48 \\
\hline Clopidogrel & $188(4.8)$ & $7(13.2)$ & 0.005 \\
\hline LMWH & $37(0.9)$ & $7(13.2)$ & $<0.001$ \\
\hline
\end{tabular}

$\mathrm{BMI}=$ Body mass index, $\mathrm{CRF}=$ Chronic renal failure, $\mathrm{LMWH}=\mathrm{Low}$ molecular weight heparin. Analyses were performed using Student's t-test or Mann-Whitney U test for between-group comparisons. Categorical variables were compared with Chi-Square or Fisher's exact test. 
Table 2. Univariate and multivariate predictors of the access site complication

\begin{tabular}{|c|c|c|c|c|}
\hline & \multicolumn{2}{|c|}{ Univariate Analysis } & \multicolumn{2}{|c|}{ Multivariate Analysis } \\
\hline & OR $(95 \%$ CI) & $p$ value & OR $(95 \% \mathrm{CI})$ & $p$ value \\
\hline Age & $0.98(0.96-1.008)$ & 0.19 & & \\
\hline Gender, female & $2.08(1.21-3.59)$ & 0.008 & $2.13(1.14-3.99)$ & 0.017 \\
\hline BMI & $0.98(0.92-1.04)$ & 0.61 & & \\
\hline Hypertension & $0.91(0.53-1.57)$ & 0.74 & & \\
\hline Diabetes mellitus & $0.90(0.50-1.61)$ & 0.73 & & \\
\hline CRF & $3.02(1.07-8.53)$ & 0.03 & $3.15(1.06-9.29)$ & 0.038 \\
\hline Systolic blood pressure & $1.003(0.99-1.01)$ & 0.68 & & \\
\hline Diastolic blood pressure & $0.99(0.97-1.01)$ & 0.67 & & \\
\hline Hemoglobin & $0.85(0.74-0.98)$ & 0.02 & $0.94(0.79-1.11)$ & 0.47 \\
\hline Platelet & $0.99(0.99-1.002)$ & 0.25 & & \\
\hline Manual compression time & $1.23(1.17-1.29)$ & $<0.001$ & $1.23(1.17-1.30)$ & $<0.001$ \\
\hline Aspirin & $1.21(0.70-2.08)$ & 0.48 & & \\
\hline Clopidogrel & $3.02(1.34-6.77)$ & 0.007 & $2.00(0.79-5.08)$ & 0.14 \\
\hline LMWH & $15.96(6.76-37.67)$ & $<0.001$ & $15.68(6.05-40.61)$ & $<0.001$ \\
\hline $\begin{array}{l}\text { Number of intervention } 1 \\
\text { versus } \geq \mathrm{c} 2\end{array}$ & $1.03(0.59-1.81)$ & 0.90 & & \\
\hline
\end{tabular}

$\mathrm{BMI}=$ Body mass index, $\mathrm{CRF}=$ Chronic renal failure, $\mathrm{LMWH}=$ Low molecular weight heparin. The access site complication predictors were determined with univariate and multivariate logistic regression analysis.

were found more frequent in ASC group. The hemoglobin level was lower and manual compression time was longer in the ASC group (Table 1). The ASCs rate were more frequent in female patients (ASC rate; male: $1 \%$ [24 / 2507] vs female: $1.7 \%$ [25 / 1465], $p$ $=0.03)$. Sheath sizes were similar in male and female patients (female; 7F / 6F: 19 [1.3\%] / 1446 [98.7\%] vs male; 7F / 6F: 42 [1.7\%] / 2465 [98.3\%], $p=0.34$ ).

In univariate logistic regression analysis female gender, presence of CRF, low hemoglobin level, use of clopidogrel and LMWH, longer manual compression time were found as predictors of ASCs (Table 2). According to multivariate logistic regression analysis female gender; OR: 2.13, 95\% CI: 1.14-3.99 ( $p=$ $0.017)$, presence of CRF; OR: $3.15,95 \%$ CI: $1.06-$ 9.29 ( $p=0.038)$, use of LMWH; OR: $15.68,95 \% \mathrm{CI}$ : 6.05-40.61 $(p<0.001)$ and manual compression time; OR: $1.23,95 \%$ CI: $1.17-1.30(p<0.001)$ were predictors of ASC (Table 2).

A ROC analysis was performed to assess the predictive power of the manual compression time for ASCs. The area under the curve was calculated 0.76 and longer manual compression time was related with ASC $(p<0.001)$ (Fig. 1). According to ROC analysis, longer than 10.5 minutes manual compression time had $62 \%$ sensitivity and $82 \%$ specificity for ASCs.

The distribution of 53 ASCs were; 6 pseudoaneurysm, 2 bleeding ( $>3 \mathrm{~g} / \mathrm{dl}$ drop in hemoglobin), 2 dissection and 43 hematoma (hematoma $>10 \mathrm{~cm}$ in diameter). Three pseudo-aneurysm and 3 big hematomas were treated by surgery, the remaining 3 pseudoaneurysm and 40 hematomas were treated by manual compression. Two dissection were treated conservatively, the 2 bleeding (one of them was retroperitoneal bleeding, the other patient bleed out of the groin because sand bag was not correct position) were treated with blood transfusion.

\section{DISCUSSION}

The principal findings of the present study are as follows: 1) the femoral ASC rate was $1.3 \%, 2$ ) female gender, presence of CRF, LMWH using and longer 


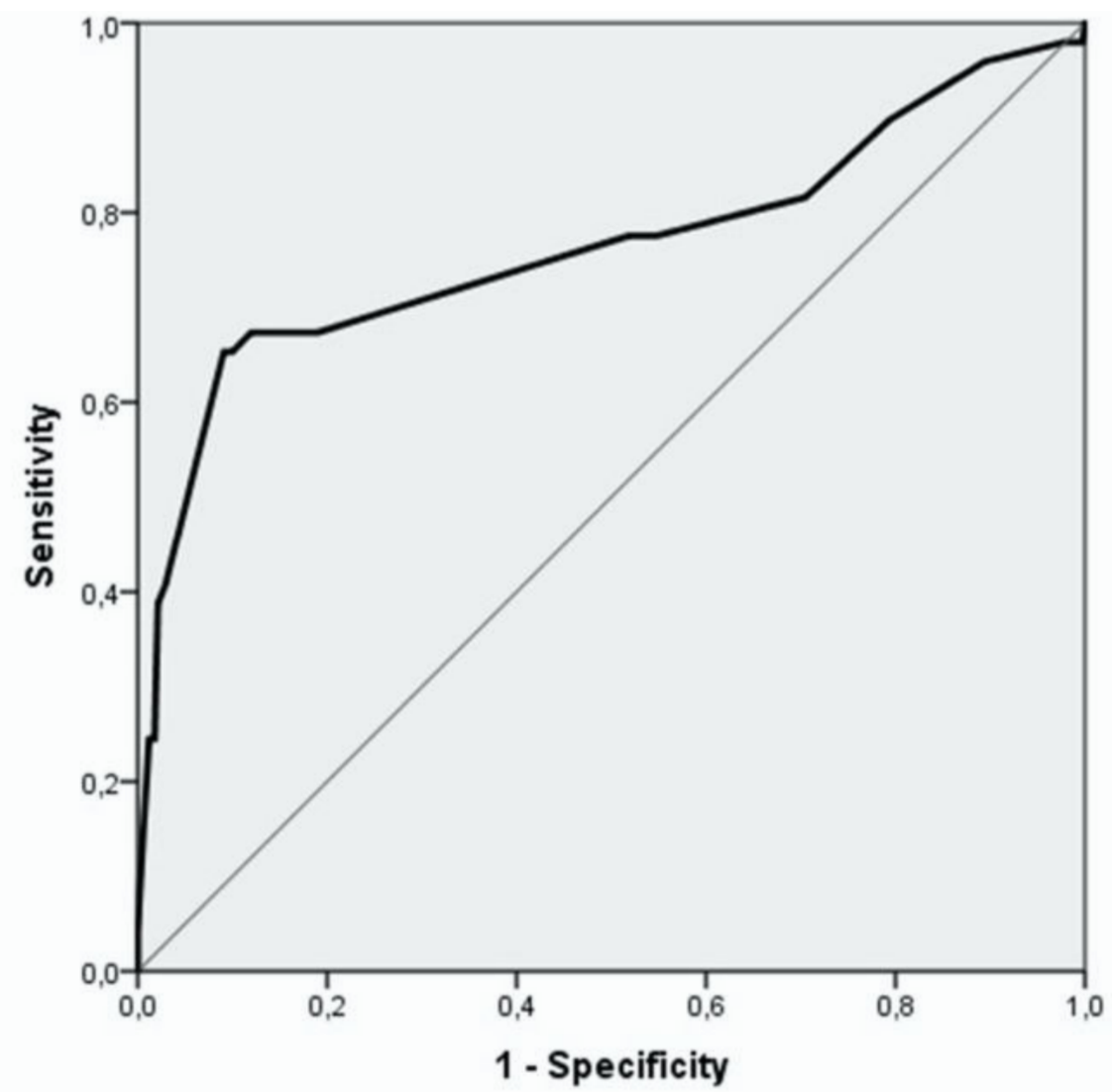

Fig. 1. ROC analysis between the manual compression time and access site complication.

manual compression time were predictors of femoral ASC.

ASC remains an important and common adverse events after DCA and could be concluded with increased risk of morbidity, prolonged duration of hospitalization and procedural costs. In recent years, catheter technologies have improved. So that ASC rate may become lower. According to these knowledge, we decided to assess femoral ASC rate and determine the predictors of ASC after DCA in our experience. In our population (3972 consecutive patients) we performed DCA via TFA, the incidence of ASC rate was $1.3 \%$. The incidence of ASC rate was consistent with recent studies. While 7-8 F catheters were used in the 1980s, DCA can now be performed using 5-6 F catheters [11]. Over the years, the operator experience has increased, and we enrolled the study only DCA patients. Because of these factors the ASC rate was as low as $1.3 \%$.

In the RIVAL trial, major vascular access complication rate was $3.7 \%$ in the femoral group [12]. However, the RIVAL study population consisted of patients with acute coronary syndrome. The study population was treated with anticoagulant and antiagregan agent. In an other study of acute coronary syndrome, the incidence of entry site complications were found $7.5 \%$ [13]. The ASC rate was nearly $5 \%$, pseudoaneurysm and hematoma $>5 \mathrm{~cm}$ were seen in $1 \%$ and $1.5 \%$ rate respectively [13]. Low rates of ASC in our study compared to the acute coronary syndrome trials could be explained with study population and medication differences (antiagregan and anticoagulant treatment). Our study population consisted with only DCA patients.

The previous studies demonstrated that risk factors for femoral access complications include female gender, over- and underweight, older age, uncontrolled hypertension, previous catheterization at the same site, high level of anticoagulation, larger arterial sheaths, renal failure, concomitant venous sheath, prolonged sheath duration time $[14,15]$. Yatskar et al. [16] also showed similar results in their study female sex, concomitant renal disease were significantly associated with access site hematoma requiring transfusion. The incidence of hematoma requiring transfusion was $1.8 \%$ and also they found that glyco- 
protein IIb/IIIa inhibitors, thrombolytic therapy, and post procedure heparin were more commonly used in hematoma requiring transfusion patients [17]. And also, in our study we demonstrated that the incidence of ASC was related with female gender, CRF, LMWH using and long manual compression time.

In this study we showed that patients who have impaired renal function could tend to be at increased risk of ASC and most of study results were consistent with our study $[17,18]$. In renal insufficiency patients, the complications such as hematomas and pseudo aneurysms could be seen more frequently because the hemostatic functions are impaired. The cause of the hemorrhage is platelet dysfunction due to guanidine succinic acid accumulation. This substance causes platelet factor 3 release, platelet adherence and aggregation disorders, and thereby prolonging bleeding time. Likewise the ASC rate was higher with LMWH using, because of the anticoagulant effect.

Female gender was related with higher incidence of ASC, previous studies showed that female gender was an independent predictor of ASC $[19,20]$. The differences with gender could be explained with the ratio of sheath size to access artery. The female patients femoral artery are smaller than male patients [21]. The sheath size is an independent predictor of ASC. We use the same size sheath in female and male patients but the ratio of sheath size to access artery is should be higher in female group than male group. The next studies could evaluate the access site femoral artery size to sheath ratio.

Manual compression time is related with the vascular hemostasis of the access site. Anatomical features of access site and coagulation features of the patients are directly affect the vascular hemostasis of the access site. Longer hemostasis time need longer manual compression. The ASC rate is increase with delayed hemostasis. Repeated interventions in the same region may alter the anatomical characteristics of that region, resulting in delayed vascular hemostasis and increased risk of complications. As a result, our study is the first study, showing that manual compression time is a predictor of ASC. Patients who need long compression times to achieve hemostasis can be followed up more carefully and complications could be prevent.

There are different methods to achieve vascular hemostasis after DCA. These methods have not supe- riority to each other. Previous studies showed that manual compression as effective as mechanical compression for achieving hemostasis and there is no real improvement in clinical outcomes with vascular closure devices when compared with manual compression $[22,23]$. The latest trial (The SAFARISTEMI trial) failed to show that radial access was superior to femoral access in primary PCI [24]. However this is a randomized trial in selected patients trial (female gender, CRF, anticoagulant using) may found superiority of the radial access.

ASC is also related to anatomic risk factors include blind puncture of femoral artery, vessel calcification, placement of venous sheath [22]. Anatomic risk factors could be modified to reduce the risk of complications by using ultrasound scan or fluoroscopy to aid in the optimal puncture site.

\section{Limitations}

First; our study population include only DCA patients so that we cannot have data about the percutaneous coronary intervention patients. Second; this study is a single center study and include only one operator team. Third we have not got femoral artery anatomical features, like as femoral artery size and calcification in our study population. Fourth, we used $6 \mathrm{~F}$ sheath for DCA nearly all patients, therefore we do not have data about smaller and larger size sheath.

\section{CONCLUSION}

Incidence of ASC related to DCA in our study was found to be similar to data throughout the world and as low as 1,3\%. Female gender, presence of CRF, LMWH using and long manual compression time were independent predictors of ASC. Operators should perform TRA or use vascular closure devices, in patients who had these risk factors to reduce ASC rate.

\section{Conflict of interest}

The authors disclosed no conflict of interest during the preparation or publication of this manuscript.

\section{Financing}

The authors disclosed that they did not receive any grant during conduction or writing of this study. 


\section{REFERENCES}

1. Rajani NK, Brown AJ, Mccormick LM, Parwaiz H, Kaushal A, Hoole SP, et al. Institutional switch from transfemoral to transradial vascular access for percutaneous coronary intervention was associated with a reduction in bleeding events: a single center experience of $>10,000$ consecutive cases. J Interv Cardiol 2015;28:296-304.

2. Fitts J, Ver Lee P, Hofmaster P, Malenka D, Group NNECS. Fluoroscopy-guided femoral artery puncture reduces the risk of PCI-related vascular complications. J Interv Cardiol 2008;21:273-8.

3. Sherev DA, Shaw RE, Brent BN. Angiographic predictors of femoral access site complications: implication for planned percutaneous coronary intervention. Catheter Cardiovasc Interv 2005;65:196-202.

4. Wongcharoen W, Pinyosamosorn K, Gunaparn S, Boonnayhun S, Thonghong T, Suwannasom P, et al. Vascular access site complication in transfemoral coronary angiography between uninterrupted warfarin and heparin bridging. J Interv Cardiol 2017;30:387-92.

5. Bhatty S, Cooke R, Shetty R, Jovin IS. Femoral vascular access-site complications in the cardiac catheterization laboratory: diagnosis and management. Interv Cardiol 2011;3:503-14. 6. Ramana RK, Singh A, Dieter RS, Moran JF, Steen L, Lewis $\mathrm{BE}$, et al. Femoral angiogram prior to arteriotomy closure device does not reduce vascular complications in patients undergoing cardiac catheterization. J Interv Cardiol 2008;21:204-8.

7. Tarighatnia A, Mohammadalian A, Ghojazade M, Pourafkari L, Farajollahi A. Beam projections and radiation exposure in transradial and transfemoral approaches during coronary angiography. Anatol J Cardiol 2017;18:298-303.

8. Bashore TM, Balter S, Barac A, Byrne JG, Cavendish JJ, Chambers CE, et al. 2012 American College of Cardiology Foundation/Society for Cardiovascular Angiography and Interventions expert consensus document on cardiac catheterization laboratory standards update: a report of the American College of Cardiology Foundation Task Force on Expert Consensus Documents. J Am Coll Cardiol 2012;59:2221-305.

9. Cockcroft DW, Gault MH. Prediction of creatinine clearance from serum creatinine. Nephron 1976;16:31-41.

10. Rao AK, Pratt C, Berke A, Jaffe A, Ockene I, Schreiber TL, et al. Thrombolysis in Myocardial Infarction (TIMI) Trialphase I: hemorrhagic manifestations and changes in plasma fibrinogen and the fibrinolytic system in patients treated with recombinant tissue plasminogen activator and streptokinase. J Am Coll Cardiol 1988;11:1-11.

11. Ammann P, Brunner-La Rocca HP, Angehrn W, Roelli H, Sagmeister M, Rickli H. Procedural complications following diagnostic coronary angiography are related to the operator's experience and the catheter size. Catheter Cardiovasc Interv 2003;59:13-8.

12. Jolly SS, Yusuf S, Cairns J, Niemelä K, Xavier D, Widimsky $\mathrm{P}$, et al. Radial versus femoral access for coronary angiography and intervention in patients with acute coronary syndromes (RIVAL): a randomised, parallel group, multicentre trial. Lancet 2011;377:1409-20.

13. Choussat R, Black A, Bossi I, Fajadet J, Marco J. Vascular complications and clinical outcome after coronary angioplasty with platelet IIb/IIIa receptor blockade. Comparison of transradial vs transfemoral arterial access. Eur Heart J 2000;21:662-7. 14. Mandak JS, Blankenship JC, Gardner LH, Berkowitz SD, Aguirre FV, Sigmon KN, et al. Modifiable risk factors for vascular access site complications in the IMPACT II trial of angioplasty with versus without eptifibatide. J Am Coll Cardiol 1998;31:1518-24.

15. Omoigui NA, Califf RM, Pieper K, Keeler G, O'Hanesian MA, Berdan LG, et al. Peripheral vascular complications in the coronary angioplasty versus excisional atherectomy trial (CAVEAT-I). J Am Coll Cardiol 1995;26:922-30.

16. Yatskar L, Selzer F, Feit F, Cohen HA, Jacobs AK, Williams $\mathrm{DO}$, et al. Access site hematoma requiring blood transfusion predicts mortality in patients undergoing percutaneous coronary intervention: data from the National Heart, Lung, and Blood Institute Dynamic Registry. Catheter Cardiovasc Interv 2007;69:961-6.

17. Waksman R, King SB, Douglas JS, Shen Y, Ewing H, Mueller L, et al. Predictors of groin complications after balloon and new-device coronary intervention. Am J Cardiol 1995;75:886-9.

18. Hanlon C, Rosenthal J. The Pennsylvania Learning Exchange: Helping states improve and integrate patient safety initiatives. Summary report. New York: The Commonwealth Fund; 2007.

19. Eslami MH, Csikesz N, Schanzer A, Messina LM. Peripheral arterial interventions: trends in market share and outcomes by specialty, 1998-2005. J Vasc Surg 2009;50:1071-8.

20. Applegate RJ, Sacrinty MT, Kutcher MA, Kahl FR, Gandhi SK, Santos RM, et al. Trends in vascular complications after diagnostic cardiac catheterization and percutaneous coronary intervention via the femoral artery, 1998 to 2007. JACC: Cardiovasc Interv 2008;1:317-26.

21. Sandgren T, Sonesson B, Ahlgren ÅR, Länne T. The diameter of the common femoral artery in healthy human: influence of sex, age, and body size. J Vasc Surg 1999;29:503-10.

22. Sulzbach-Hoke LM, Ratcliffe SJ, Kimmel SE, Kolansky DM, Polomano R. Predictors of complications following sheath removal with percutaneous coronary intervention. J Cardiovasc Nurs 2010;25:E1-E8.

23. Byrne RA, Cassese S, Linhardt M, Kastrati A. Vascular access and closure in coronary angiography and percutaneous intervention. Nat Rev Cardiol 2013;10:27.

24. Le May MR, Wells GA, So DY, Chong AY, Froeschl M, Dick A, et al. The American College of Cardiology Annual Scientific Session (ACC 2019), New Orleans, LA, March 18, 2019. 